\title{
Wie Scheinmedikamente wirken
}

Zunhammer $\mathrm{M}$ et al. Placebo Effects on the Neurologic Pain Signature: A Meta-analysis of Individual Participant Functional Magnetic Resonance Imaging Data. JAMA Neurol 2018; doi:10.1001/ jamaneurol.2018.2017

Plazeboeffekte können Schmerzen lindern. Was dabei im Gehirn passiert, wollten Wissenschaftler der Medizinischen Fakultät der Universität Duisburg-Essen herausfinden. Gemeinsam mit US-Kollegen analysierten sie rund 600 MRT-Bilder, um zu verstehen, mit welchen Mechanismen Scheinarzneimittel den Schmerz reduzieren.

Das internationale Forscherteam wollte z. B. herausfinden, ob der Plazeboeffekt die Schmerzleitung im Gehirn verändert. Dies konnten sie anhand der Daten tatsächlich nachweisen, allerdings war dieser Effekt sehr klein. Er allein kann nicht für das Ausmaß des schmerzlindernden Effektes verantwortlich sein. Es müssen deshalb auch die Gehirnnetzwerke betrachtet werden, die an der kognitiven und emotionalen Schmerzverarbeitung beteiligt sind.

Die Mediziner konnten auch zeigen, dass der Plazeboeffekt deutlich anders wirkt als echte Schmerzmittel, etwa Opioide. Der größte Unterschied ist, dass Opioide die Schmerzleitung im Gehirn zehnmal stärker beeinflussen als die Plazebobehandlungen und zwar bei gleichem analgetischen Effekt.

Die Essener Studie zeigt außerdem, dass bildgebende Verfahren, wie die Magnetresonanztomographie helfen können, den Plazeboeffekt von den Effekten pharmakologischer Substanzen abzugrenzen.

Nach einer Mitteilung der Universität Duisburg-Essen 